\title{
Wave Mediated Synchronization Of Nonuniform Oscillatory Media
}

On-Uma Kheowan

Eugene Mihaliuk

Bernd Blasius

Irene Sendiña-Nadal

Kenneth Showalter

Follow this and additional works at: https://researchrepository.wvu.edu/faculty_publications

\section{Digital Commons Citation}

Kheowan, On-Uma; Mihaliuk, Eugene; Blasius, Bernd; Sendiña-Nadal, Irene; and Showalter, Kenneth, "Wave Mediated Synchronization Of Nonuniform Oscillatory Media" (2007). Faculty Scholarship. 731.

https://researchrepository.wvu.edu/faculty_publications/731 


\title{
Wave Mediated Synchronization of Nonuniform Oscillatory Media
}

\author{
On-Uma Kheowan, ${ }^{1,2}$ Eugene Mihaliuk, ${ }^{1}$ Bernd Blasius, ${ }^{3}$ Irene Sendiña-Nadal, ${ }^{4}$ and Kenneth Showalter ${ }^{1}$ \\ ${ }^{1}$ Department of Chemistry, West Virginia University, Morgantown, West Virginia 26506-6045, USA \\ ${ }^{2}$ Department of Chemistry, Mahidol University, Rama 6 Road, Bangkok 10400, Thailand \\ ${ }^{3}$ ICBM, University of Oldenburg, 26111 Oldenburg, Germany \\ ${ }^{4}$ Departamento de Física, Universidad Rey Juan Carlos, Tulipán s/n 28933 Móstoles, Madrid, Spain
}

(Received 16 June 2006; published 13 February 2007)

\begin{abstract}
We characterize the spatiotemporal evolution of a photosensitive Belousov-Zhabotinsky medium that is made up of coupled oscillatory cells with randomly distributed frequencies. The medium evolves from an initial state of multiple wave sources to a synchronized state governed by a single wave source. The synchronization occurs via a competition between the sources, which arises when the oscillators are not identical but have slightly different natural frequencies. The evolution of each cell is monitored to demonstrate frequency and phase synchronization of the inhomogeneous cellular medium, and a simple kinematic description for the advance of the phase-diffusion wave is presented.
\end{abstract}

Self-sustained oscillators that are coupled by diffusion are found in many natural and man-made systems. A wide range of dynamics can be found in arrays of coupled oscillators, and synchronization in such systems has been extensively studied [1-3]. Several recent experimental studies have examined systems of coupled oscillators and extended oscillatory media [4-6]. The transition from oscillatory to traveling wave behavior is often observed in spatially distributed systems and is thought to play a role in certain biological [7] and ecological systems [8]. The simplest case of this transition is when a pacemaker, consisting of an oscillatory source with a shorter period than the rest of the medium, generates a pulse train that spreads into the medium [1]. Related behavior has been observed in spatially distributed chemical media such as the BelousovZhabotinsky (BZ) reaction [9], where heterogeneities such as adventitious dust particles give rise to oscillatory sources. Waves that emanate from these pacemakers propagate into the surrounding excitable medium, with the highest frequency source ultimately entraining the system.

The transition to a final coherent state imposed by the highest frequency wave source has been theoretically investigated in oscillatory media $[1,10,11]$. Kuramoto [1] showed that when two wave sources with different frequencies compete, the slower source is overtaken by the faster source, such that all oscillations eventually occur at the higher frequency. These results have been extended to the case of $n$ sources of two different frequencies [10] as well as to fully disordered systems [11].

In this Letter, we report on a systematic study of wave mediated synchronization of oscillatory cellular media in experiments and corresponding simulations. Our system is an array of oscillatory cells with controllable coupling at the cell boundaries. The cellular system differs from a system with point sources in that each cell making up the medium has a randomly assigned oscillatory frequency and is directly coupled to its nearest neighbors.
Experiments were carried out with the photosensitive $\mathrm{BZ}$ reaction [12], in which the $\mathrm{Ru}(b p y)_{3}^{2+}$ catalyst is immobilized in a gel [13]. The reaction mixture composition was prepared so that under dark conditions the medium was oscillatory. An illumination pattern consisting of an array of squares separated by boundaries of higher intensity illumination was projected onto the gel. This pattern produced a lattice of oscillatory cells coupled through regions of lower excitability. Each experiment was initiated in two phases. The dynamical behavior was first reset by applying high intensity light uniformly across the medium, rendering the entire system nonexcitable and erasing all previous activity. The static illumination pattern was then imposed, with the period of each oscillator determined by the local light intensity. Random light intensities were uniformly distributed with maximal disorder over the lattice such that all cells remained in the oscillatory regime.

The transition to the synchronized state is shown in Fig. 1, where the instantaneous period of each cell is plotted as a function of time. The periods are initially distributed according to the variation in light intensity and differ over the range $T_{i}=25.14 \pm 2.15 \mathrm{~s}$ (standard deviation). The transient behavior involves smooth changes of the periods, interrupted by abrupt jumps as the system evolves. Several synchronized clusters can be seen, and the system gradually evolves so that all cells are eventually entrained to a common frequency. A gradual increase in average period is observed in Fig. 1 due to the experimental drift of the BZ system; however, all cells eventually synchronize to the highest frequency cell. We note that similar frequency changes, including abrupt, punctuated jumps, have been theoretically characterized in distributed oscillatory media [10].

The spatiotemporal evolution of the pattern in a typical experiment is shown in Fig. 2, with a space-time plot in the left panel and corresponding snapshots of the wave behav- 


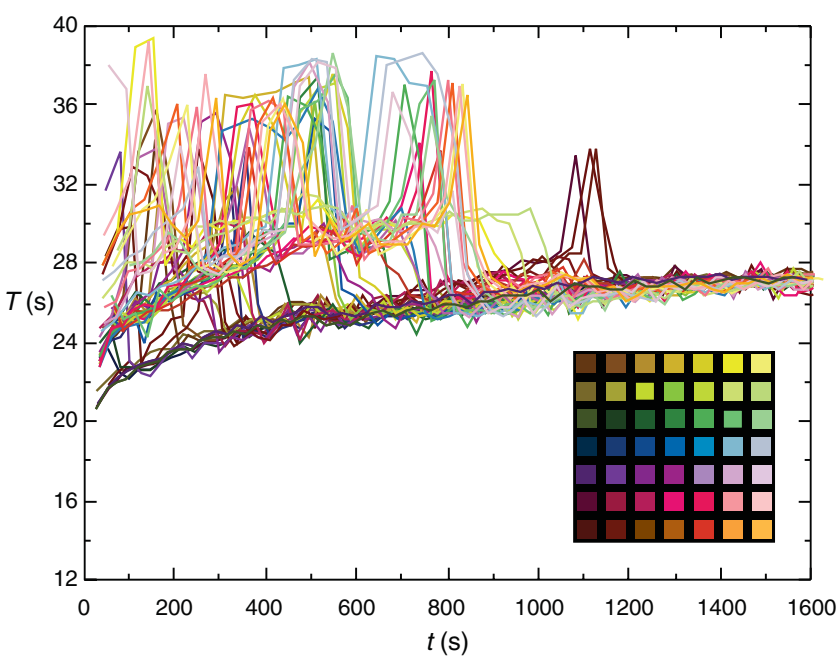

FIG. 1 (color). Transition to synchronization in a square lattice of $7 \times 7$ cells in a photosensitive Belousov-Zhabotinsky (BZ) medium. The instantaneous period of oscillation of each cell is plotted as a function of time, and the time traces are color coded with respect to the corresponding cells (shown in inset). The ruthenium-loaded gel was enclosed in a reactor with a continuous flow of catalyst-free $\mathrm{BZ}$ solution, with $\left[\mathrm{NaBrO}_{3}\right]=0.4 \mathrm{M}$, $[$ malonic acid $]=0.2 \mathrm{M}, \quad\left[\mathrm{H}_{2} \mathrm{SO}_{4}\right]=0.7 \mathrm{M}$, and $[\mathrm{NaBr}]=$ $0.125 \mathrm{M}$. The $0.28 \mathrm{~mm}$ thick gel contained $2.4 \mathrm{mM}$ $\mathrm{Ru}(b p y)_{3}^{2+}$. The residence time of the reaction mixture was $295 \mathrm{~s}$, and the system was maintained at $22 \pm 1^{\circ} \mathrm{C}$. The light intensity of each cell $(1.65 \mathrm{~mm} \times 1.65 \mathrm{~mm})$ was determined by a uniform distribution function between 0.008 and $0.472 \mathrm{~mW} \mathrm{~cm}^{-2}$, and the intensity was $2.0 \mathrm{~mW} \mathrm{~cm}^{-2}$ in the boundary $(0.8 \mathrm{~mm}$ wide $)$ around each cell.

ior to the right. We see an initial state $(t=50 \mathrm{~s})$ with independent oscillations in each cell, where an expanding wave forms at the center of each cell. These waves propagate outward until they collide with waves generated by neighboring cells. Eventually, only two cells with nearly the same frequency dominate the entire system, as shown in the last snapshot in Fig. $2(t=1250 \mathrm{~s})$. The competition between the different pacemakers can also be seen in the space-time plot, which is taken along the horizontal cut indicated in the first snapshot. This competition defines collision boundaries in the phase profile, shown by the nearly vertical dotted lines in the space-time plot, where waves from different domains collide.

The far right-hand panels in Fig. 2 show the phase difference of each cell relative to an arbitrary reference cell, where each panel corresponds to the snapshot to the left. The phase differences vary as the system evolves until the entire medium becomes entrained by the phasediffusion wave, whereupon the phase differences become stationary. This phase synchronization arises from the phase-diffusion wave, and therefore, the phase is a function of distance from the wave source: $\phi(r, t) \approx \omega t-k r$, where $r=\left|\mathbf{r}-\mathbf{r}_{0}\right|$ is the distance from the wave source $\mathbf{r}_{0}, \omega$ is the oscillation frequency, and $k$ is the wave number [14]. Since the time evolution of all phases is the same in

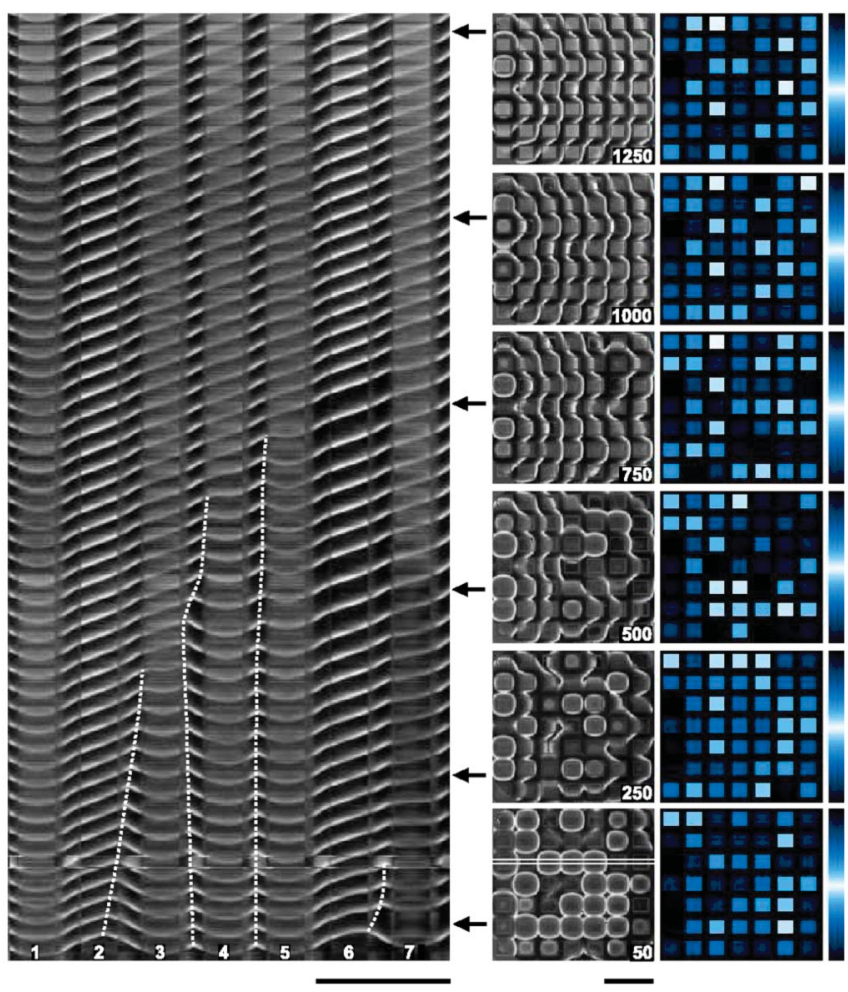

FIG. 2 (color). Evolution of spatial patterns in an array of cells with a distribution of oscillatory frequencies. The middle panel shows snapshots of the pattern evolution, where time (in seconds) is indicated in the lower right-hand corner of each snapshot. The left panel shows a space-time plot taken along the horizontal cut indicated in the first snapshot $(t=50 \mathrm{~s})$. Dotted lines in the space-time plot depict the collision boundaries, where waves from different domains collide. Numbers at the bottom of the left panel indicate the cell number. Arrows between the panels show the time in the space-time plot of each snapshot. The right panel shows the successive phase difference (taken as modulo $2 \pi$ ) between each cell relative to an arbitrary reference cell (left column, third row) [15]. Scale bar: $5 \mathrm{~mm}$; phase color coded by bar on right. Data and images are from the experiment in Fig. 1. (See movie in Ref. [27]).

the synchronized state, we can define a phase profile as the difference of the phases at each point with respect to the phase at an arbitrary reference point, $\theta(r, t) \approx \phi(r, t)-$ $\phi\left(r_{\text {ref }}, t\right)[15]$. This phase profile is stationary, as shown by the V shape in the space-time plot after $t \approx 1000 \mathrm{~s}$, which corresponds to the phase-diffusion wave.

Figure 3 shows the evolution of a system identical to that in Fig. 2 except that the cell boundaries were made much less excitable by imposing higher light intensity in these regions. After the initial transient behavior, shown in the first snapshot, the oscillations in the cells occur independently, as can be seen in the subsequent snapshots. The light intensity distribution gives rise to some cells with higher excitability that oscillate frequently, while other cells with lower excitability oscillate infrequently or not at all [16]. With the nonexcitable boundaries, virtually no interaction occurs between the oscillators and there is no 


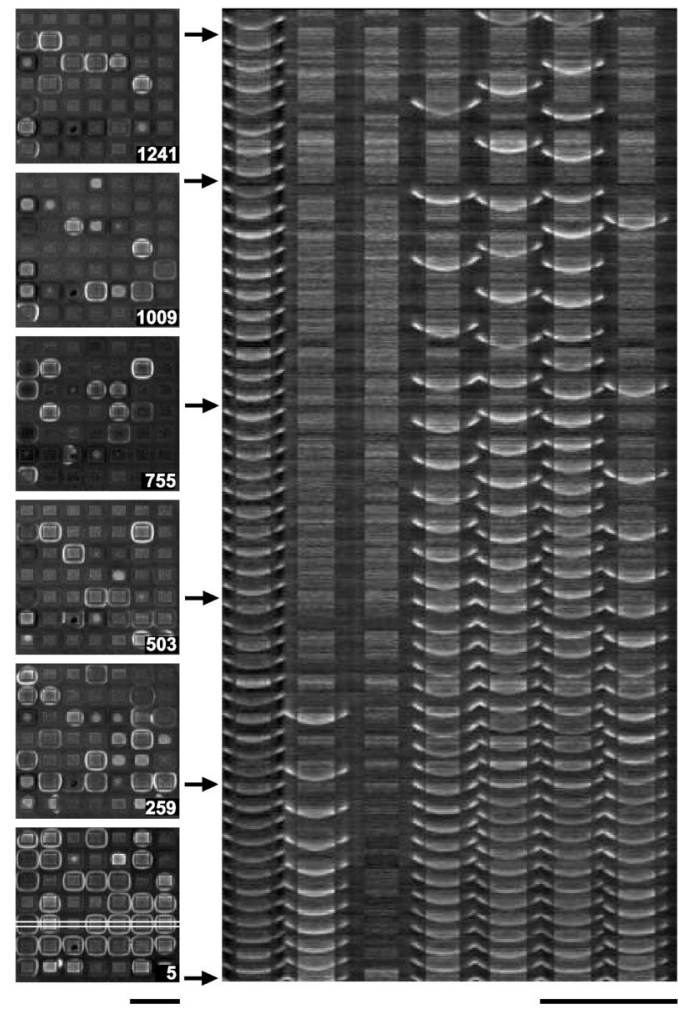

FIG. 3. Evolution of an array of independent oscillators. All experimental conditions are the same as described in Figs. 1 and 2 except higher light intensity at the cell boundaries. Snapshots (left) show the pattern evolution; the space-time plot (right) was taken along the horizontal cut indicated in the first snapshot $(t=$ $5 \mathrm{~s}$ ). Arrows between the panels show the time in the space-time plot of each snapshot. The light intensity was $3.8 \mathrm{~mW} \mathrm{~cm}^{-2}$ in the boundary around each cell. Scale bar: $5 \mathrm{~mm}$. (See movie Ref. [27]).

synchronization, as can be seen in the space-time plot to the right.

Simulations of wave mediated synchronization were carried out with the Oregonator model of the photo-BZ reaction [17] in an analogous array of cells, with each cell surrounded by a lower excitability boundary. Figure 4(a) shows a space-time plot and snapshots of a $7 \times 7$ cell array in which the excitability was varied in each cell by assigning a random value to the parameter $\Phi$ in the model, corresponding to the illumination intensity $[12,13]$. The first and second snapshots reveal a pacemaker emerging at a cell (row 5, column 2) that develops into a target pattern in subsequent snapshots. The space-time plot shows the evolution of the synchronization by this wave source, which appears as the global minimum in each phase profile, as the other sources become entrained.

Insights into the synchronization can be gained by examining two neighboring cells having slightly different oscillatory frequencies, as shown in the space-time plot in Fig. 4(b). The two cells begin with the same phase; however, there is an incremental advance in phase of the left cell with respect to the right cell with each oscillation

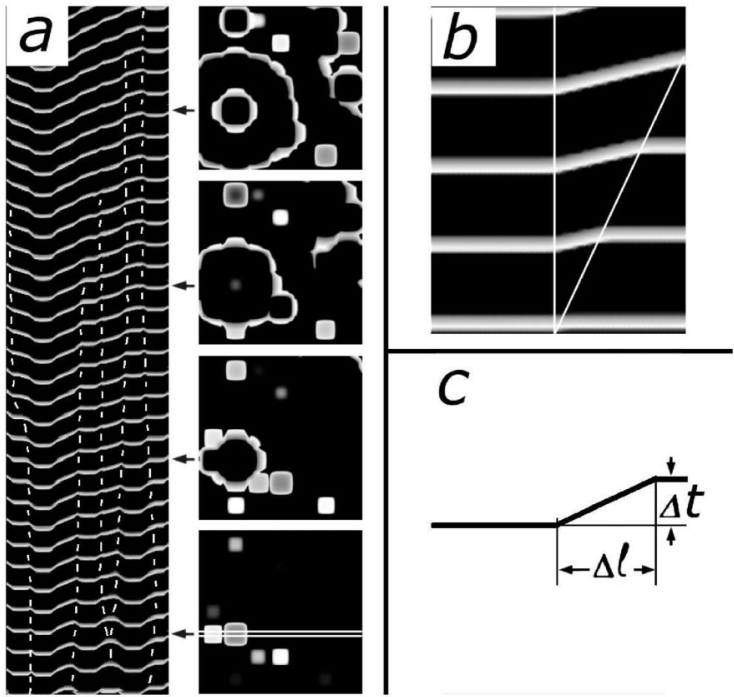

FIG. 4. Numerical simulations of a square lattice of $7 \times 7$ oscillatory cells [17]. (a) Snapshots (right) of 2D system and the corresponding space-time plot (left) for the 1D cross section indicated in the first snapshot. Arrows indicate times of the snapshots in the space-time plot. The value of $\Phi$ in each cell $(26 \times 26$ grid points) was determined by a uniform distribution function between 0.0 and 0.007 , and the value was 0.05 in the boundary ( 9 grid points wide) around each cell. (b) Competition between two oscillatory cells, where $\Phi_{L}=0.0$ and $\Phi_{R}=0.004$ in the left and right cells, respectively. (c) Definition of parameters.

due to the higher frequency of the left cell. The left and right extremes of the medium oscillate virtually independently; however, at the point of contact between the cells, there is a gradual transition from homogeneous oscillation to a traveling wave.

The time from the initially independent behavior, with zero phase shift, to the complete entrainment can be estimated by observing that the slope of the traveling wave in the space-time plot corresponds to the inverse of the wave velocity $\mathrm{v}$. At the same time, this slope is equal to the ratio of the wave propagation distance $\Delta l$ to the delay $\Delta t$ between the left and right cells, $\frac{\Delta l}{\Delta t}=\mathrm{v}$, as shown in Fig. 4(c). The delay accumulates linearly from the initiation time and increments by the period difference between the left and right cells $\left(T_{1}-T_{0}\right)$ on each oscillation, $\Delta t=\frac{t}{T_{0}}\left(T_{1}-T_{0}\right)$. Together, these relationships give the time to entrainment, $t=\tau$, as proportional to the cell size, $\Delta l=L$, and the smaller oscillation period $T_{0}$, and inversely proportional to the traveling wave velocity and the difference between the periods:

$$
\tau=\frac{L T_{0}}{\mathrm{v}\left(T_{1}-T_{0}\right)} .
$$

For small differences in excitability, the wave velocity is virtually constant (see Figs. 2 and 4), and the most significant factor in the competition between the cells is the difference between their natural frequencies. 
We can apply this analysis to determine the time required for the successive entrainment of cells, such as the entrainment of cell 3 by cell 2 , cell 4 by cell 3 , and cell 5 by cell 4 in row 3, shown in Fig. 2 (see horizontal cut in snapshot at $t=50 \mathrm{~s}$ ). With the measured period of each cell, the wave velocity $\mathrm{v}=0.067 \mathrm{~mm} \mathrm{~s}^{-1}$ and cell width $L=1.65 \mathrm{~mm}$, Eq. (1) gives entrainment times of 132, 188, and $88 \mathrm{~s}$ compared to the measured entrainment times of 140, 170, and $97 \mathrm{~s}$. While the agreement between the predicted and measured entrainment times is good, close inspection of the entrainment reveals phase wave behavior that is more complex than can be accounted for by the simple kinematic description of Eq. (1), since the oscillations in each cell do not occur uniformly.

Collision boundaries between competing spiral waves, recently characterized theoretically $[21,22]$ and experimentally [23], are directly relevant to our kinematic description, Eq. (1). Recent studies concerning synchronization rate as a function of system size in arrays of relaxation oscillators [24] and the treatment of an oscillator chain driven by a pacemaker [1], which has been further developed for certain classes of coupling [25], are also relevant to our study. The effects of natural frequency dispersion are likely to play an important role in population dynamics [8] and in neural systems [26].

We thank the National Science Foundation (No. CHE0415392) and the W.M. Keck Foundation for supporting this research. B. B. was supported by the VolkswagenStiftung.

[1] Y. Kuramoto, Chemical Oscillations, Waves, and Turbulence (Springer-Verlag, Berlin, 1984).

[2] A. Pikovsky, M. Rosenblum, and J. Kurths, Synchronization. A Universal Concept in Nonlinear Sciences (Cambridge University Press, Cambridge, 2001).

[3] S.C. Manrubia, A.S. Mikhailov, and D.H. Zanette, Emergence of Dynamical Order: Synchronization Phenomena in Complex Systems (World Scientific, Singapore, 2004).

[4] W. Wang, I. Z. Kiss, and J. L. Hudson, Phys. Rev. Lett. 86, 4954 (2001).

[5] G. Bub, A. Shrier, and L. Glass, Phys. Rev. Lett. 94, 028105 (2005).

[6] V. K. Vanang, L. Yang, M. Dolnik, A. M. Zhabotinsky, and I. R. Epstein, Nature (London) 406, 389 (2000).

[7] A. T. Winfree, The Geometry of Biological Time (Springer, New York, 1980).

[8] B. Blasius, A. Huppert, and L. Stone, Nature (London) 399, 354 (1999).

[9] A. N. Zaikin and A.M. Zhabotinsky, Nature (London) 225, 535 (1970).

[10] A. S. Mikhailov and A. Engel, Phys. Lett. A 117, 257 (1986).
[11] B. Blasius and R. Tönjes, Phys. Rev. Lett. 95, 084101 (2005).

[12] L. Kuhnert, Nature (London) 319, 393 (1986).

[13] S. Kádár, J. Wang, and K. Showalter, Nature (London) 391, 770 (1998).

[14] The low-excitability boundary around each cell and the excitability distribution over the array of cells make this relation an approximation. The largest perturbation arises from the cell boundaries, which can be seen as "wiggles" in the V-shaped phase profile.

[15] The phase at time $t$ was determined in each cell every $0.5 \mathrm{~s}$ according to $\phi=\left(\frac{t-t_{1}}{t_{2}-t_{1}}\right) 2 \pi$, where $t_{1}$ and $t_{2}$ designate times at the beginning and the end of one period of oscillation measured at a specific amplitude of light intensity. The phase difference $\theta(r, t)$ is the difference between the phase of each cell and an arbitrary reference cell (column 1, row 3 ). The accuracy of the phase difference is dependent on the measurements of $t_{1}$ and $t_{2}$, which are accurate to within approximately $2 \%$.

[16] The high light intensity of the cell boundaries causes the experimental drift in the period to be accentuated and the cells with the lowest excitabilities to become nonoscillatory.

[17] Numerical studies were carried out using an Oregonator model of the photosensitive BZ reaction [18-20]: $\frac{\partial u}{\partial t}=$ $D_{u} \nabla^{2} u+\frac{1}{\epsilon}\left[u-u^{2}-(\Phi+f v) \frac{u-q}{u+q}\right], \frac{\partial v}{\partial t}=u-v$, where $u$ and $v$ are the dimensionless concentrations of $\mathrm{HBrO}_{2}$ and $\mathrm{Ru}(b p y)_{3}^{3+}$, respectively, $D_{u}$ is the diffusion coefficient of $\mathrm{HBrO}_{2}, \nabla^{2}$ is the Laplacian operator, and $\Phi$ represents the rate of bromide production from the irradiation. Values of $\Phi$ between 0 and 0.008 produce oscillatory media, while excitability persists up to $\Phi=0.113$. An explicit Euler method was used for the numerical integration, with $f=0.7, q=0.002, \epsilon=0.03, D_{u}=1.0$, $d x=0.5$, and $d t=0.001$, where $\epsilon$ and $q$ are scaling parameters and $f$ is an adjustable stoichiometric parameter.

[18] R. J. Field and R. M. Noyes, J. Chem. Phys. 60, 1877 (1974).

[19] J. J. Tyson and P. C. Fife, J. Chem. Phys. 73, 2224 (1980).

[20] H. J. Krug, L. Pohlmann, and L. Kuhnert, J. Phys. Chem. 94, 4862 (1990).

[21] M. Hendrey, E. Ott, and T. M. Antonsen, Jr., Phys. Rev. Lett. 82, 859 (1999).

[22] M. Hendrey, E. Ott, and T. M. Antonsen, Jr., Phys. Rev. E 61, 4943 (2000).

[23] L. B. Smolka, B. Marts, and A. L. Lin, Phys. Rev. E 72, 056205 (2005).

[24] S. R. Campbell, D. L. Wang, and C. Jayaprakash, IEEE Trans. Neural Netw. 15, 1027 (2004).

[25] N. Kopell, G. B. Ermentrout, and T. L. Williams, SIAM J. Appl. Math. 51, 1397 (1991).

[26] B. Ermentrout, J. W. Wang, J. Flores, and A. Gelperin, J. Comp. Neurol. 17, 365 (2004).

[27] See EPAPS Document No. E-PRLTAO-98-059707 for movies of Figs. 2 and 3. For more information on EPAPS, see http://www.aip.org/pubservs/epaps.html. 